\title{
Effective Elastic Modulus of Underfill Material for Flip-Chip Applications
}

\author{
Jianmin Qu and C. P. Wong, Fellow, IEEE
}

\begin{abstract}
In this paper, a micromechanics model based on the Mori-Tanaka method was developed to estimate the elastic modulus of underfill materials. An explicit expression of the underfill modulus was derived as a function of filler content and the properties of the matrix and the fillers. Predictions of the modulus from this theory were compared with experimentally measured values. Excellent agreement was observed.
\end{abstract}

Index Terms-Effective elastic modules, flip-chip, micromechanics, underfills.

\section{INTRODUCTION}

$\mathbf{M}$ OST underfill materials are two phase composites, e.g., epoxy matrix filled with ceramic particles. The primary purpose of loading ceramic particles is to reduce the coefficient of thermal expansion (CTE) and to increase the elastic modulus. These two thermal mechanical properties are critical parameters to the thermomechanical reliability of a flip-chip package. Developing underfill materials with desired values of the CTE and elastic modulus is a key enabling technology for the next generation low-cost, high-reliability flip-chip packages. In this paper, a micromechanics model is developed to estimate the elastic modulus of underfill materials based on the properties of the matrix and the fillers. To validate the prediction from the micromechanics model, samples of underfill materials with various filler compositions are made. The moduli of these samples are measured using a thermal mechanical analyzer system (TMA). Satisfactory results are obtained between the theoretically predicted and experimentally measured values.

\section{UNDERFILL FOR FLIP-CHIP APPLICATIONS}

The use of organic printed wiring boards (PWBs) as substrates in flip-chip direct chip attach (DCA) introduces a new concern: the coefficient of thermal expansion (CTE) of the PWB is much greater than that of the silicon chip. Under processing or operating conditions, the extreme thermal mismatch between the silicon integrated circuit (IC) and the organic substrate subjects the solder joints to extremely large strains, leading to early failure of the solder connections. To enhance the fatigue life and increase reliability, a rigid encapsulation layer between the chip

Manuscript received December 1, 2000; revised March 5, 2001. This work was supported by the Packaging Research Center, Georgia Tech. This work was recommended for publication by Associate Editor R. Chanchani upon evaluation of the reviewers' comments.

J. Qu is with the George W. Woodruff School of Mechanical Engineering, Georgia Institute of Technology, Atlanta, GA 30332-0405 USA.

C. P. Wong is with the School of Materials Science and Engineering, Packaging Research Center, Georgia Institute of Technology, Atlanta, GA 30332-0405 USA.

Publisher Item Identifier S 1521-3331(02)00683-9.

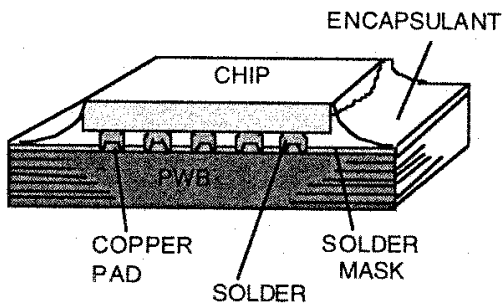

Fig. 1. Flip-chip with underfill.

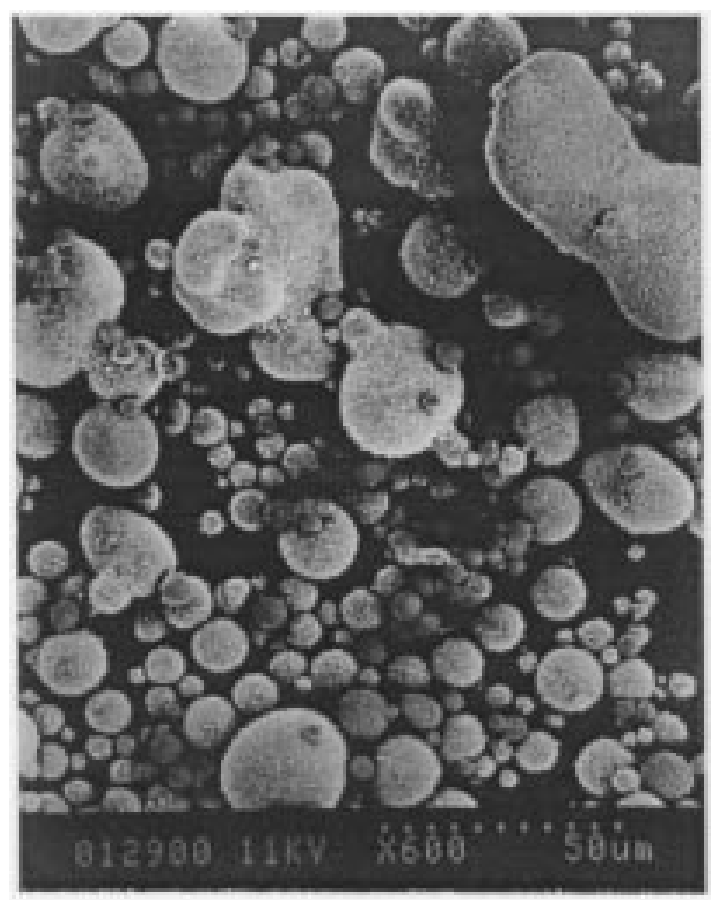

Fig. 2. Morphology of a typical silica-filled underfill material.

and the substrate encompassing the solder joints has been introduced, see Fig. 1. The encapsulant, once cured, mechanically couples the silicon and substrate together to locally constrain the CTE mismatch, and to provide a protective barrier layer on the IC chip's active surface.

Most of the underfill materials used today are two-phase composite materials. The matrix is usually an epoxy based polymer material. Silica particles are commonly used as a filler to change the modulus and CTE. Showing in Fig. 2 is a micrograph of the cross section of an epoxy matrix with silica fillers.

The introduction of underfill encapsulation can dramatically extend the fatigue life of flip chip DCA assemblies [1]. However, selection of an underfill material for a particular application is not an easy task. It has been shown [2] that there is 


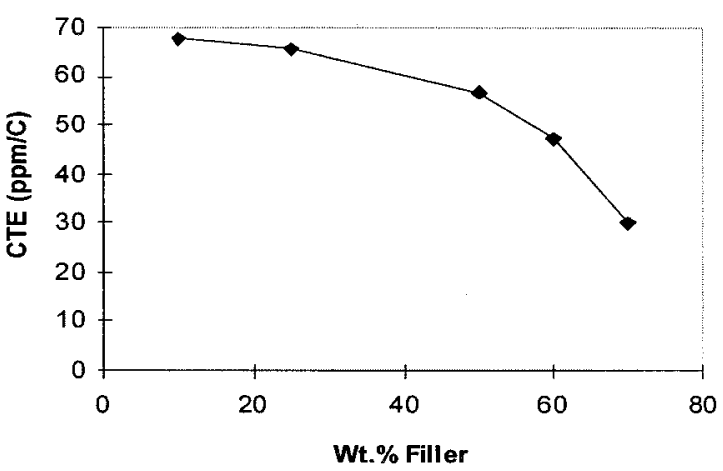

Fig. 3. CTE as function of filler loading $(15 \mu \mathrm{m}$ average particle size used for each sample).

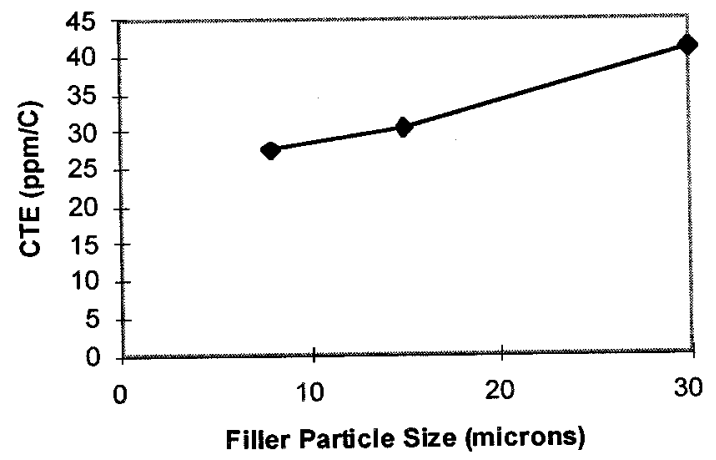

Fig. 4. CTE as function of filler particle size at Wt. $70 \%$.

an optimal range for the underfill modulus within which the stress/strain in the silicon chip and solder joints are properly optimized. It is well-known that the function of the underfill is to reduce the solder strain by mechanically coupling the die and the PWB. The stiffer the underfill is, the more the solders will be protected from fatigue failure. As the underfill modulus increases, however, more stresses are re-distributed to the die, which results in more die cracking. Therefore, it is critical to know the optimal underfill modulus such that the solders are protected just enough to satisfy the reliability requirement, while the amount of stresses transferred to the die is not large enough to cause significant die cracking.

Making use of the analysis and finite element modeling presented in [2], one can estimate the optimal value for the underfill modulus for a given package design. The next task is to develop an underfill that has the desired modulus by using the proper amount of fillers. This is easier said than done [3]. At the present time, a predictive tool is not available so the process has to be carried out on a trial and error basis. As such, it is tedious, time consuming and lacks scientific approach. For example, the neat epoxy resin/hexahydromethylphalic anhydride (HMPA) hardener mixture has a CTE of $87 \mathrm{ppm} /{ }^{\circ} \mathrm{C}$ and the silica has a CTE of $0.5 \mathrm{ppm} /{ }^{\circ} \mathrm{C}$. As the ratio of filler to resin increases, the CTE will decrease due to the lower CTE of the filler compared to that of the resin. This decrease can be seen in Fig. 3 which shows $\mathrm{CTE}$ as a function of filler loading. This graph also shows that the effect of a unit change in loading at high loadings decreases CTE more than a unit change in loading at smaller loadings. Fig. 4 shows the relationship between the CTE and the filler particle size. It is seen that as the filler particle size decreases (at a constant particle loading), the CTE also decreases. This can be explained by looking at the total surface area of the filler particles. As shown before, the CTE of the filler is much lower than that of the resin. The interface of the filler particles and the resin matrix constrict the expansion of the matrix. As the surface area of the filler particles increases due to the decrease in particle size, there is an increase in the interface between filler and resin. Therefore, an increase in the constriction of the matrix due to increased surface area allows a decrease in the expansion of the matrix [4].

In what follows, an analytical formula will be developed that enables us to calculate the effective elastic modulus of the underfill based on the moduli of the matrix and the filler materials and the filler content.

\section{THE MORI-TANAKA METHOD}

The underfill can be viewed as a composite material consisting of a matrix phase and a particle phase. Both the epoxy based matrix and silica particles can be assumed as linearly elastic and isotropic solids. Furthermore, the silica particles can be assumed spherical in shape (see Fig. 2).

Let the Young's modulus and the Poisson's ratio of the matrix be $E_{0}$ and $\nu_{0}$, respectively, and that of the particles be $E_{1}$ and $\nu_{1}$. Furthermore, assume that the volume fraction of the particles in the composite is $c$. Then, by using the Mori-Tanaka method [5], one can derive a formula for the effective Young's modulus of the composite [6]

$$
\bar{E}=2 \bar{\mu}\left[1+\frac{3 \bar{K}-2 \bar{\mu}}{2(3 \bar{K}+\bar{\mu})}\right]
$$

where

$$
\begin{aligned}
\bar{K} & =K_{0}\left\{1+\frac{c\left(K_{1}-K_{0}\right)}{K_{0}+3 \gamma_{0}(1-c)\left(K_{1}-K_{0}\right)}\right\} \\
\bar{\mu} & =\mu_{0}\left\{1+\frac{c\left(\mu_{1}-\mu_{0}\right)}{\mu_{0}+2 \delta_{0}(1-c)\left(\mu_{1}-\mu_{0}\right)}\right\} \\
\gamma_{0} & =\frac{1+V_{0}}{9\left(1-V_{0}\right)} \\
\delta_{0} & =\frac{4-5 V_{0}}{15\left(1-V_{0}\right)} \\
K_{n} & =\frac{E_{n}}{3\left(1-2 \nu_{n}\right)}, \quad n=0,1 \\
\mu_{n} & =\frac{E_{n}}{2\left(1+\nu_{n}\right)}, \quad n=0,1 .
\end{aligned}
$$

where as, $F=$ effective bulk modulus and $\bar{\mu}=$ Eff. shear modulus.

It is seen from the above equations that the modulus of the underfill is determined by the moduli of the epoxy matrix and the filler particles, as well as the particle volume fraction. Once these parameters are given, (1) can be used as a tool to estimate the Young's modulus of the underfill. On the other hand, (1) can also be used to select the matrix and particle materials and determine the amount of particles needed yield the desired modulus 


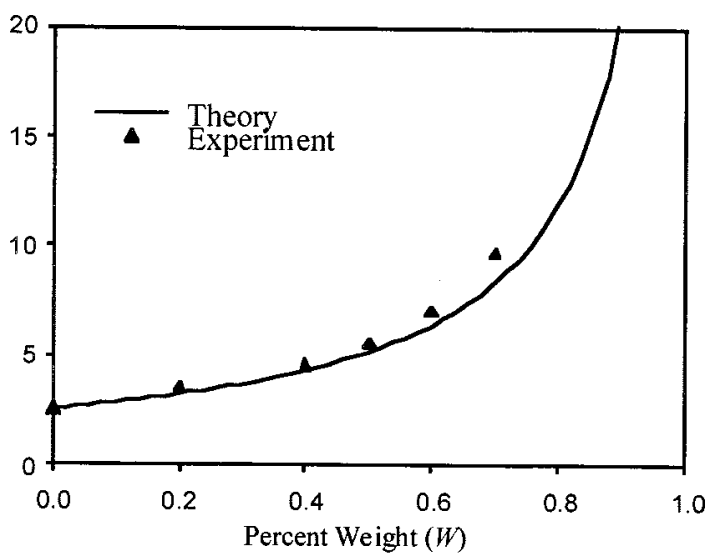

Fig. 5. Comparison between the theoretical prediction and experimental measurement.

TABLE I

MATERIAL CONSTANTS

\begin{tabular}{c|c|c|c}
\hline & Mass Density & Young's Modulus & Poisson's Ratio \\
\hline Epoxy & $\rho_{0}=1.16 \mathrm{~g} / \mathrm{cc}$ & $E_{0}=2.5 \mathrm{GPa}$ & $v_{0}=0.4$ \\
\hline Silica & $\rho_{1}=2.2 \mathrm{~g} / \mathrm{cc}$ & $E_{1}=74 \mathrm{GPa}$ & $v_{1}=0.19$ \\
\hline
\end{tabular}

for the underfill. This will greatly reduce the amount of trial and error in developing the underfill material.

Instead of the filler volume fraction, $c$, for measuring the amount of fillers, another convention is to use the percent weight, $W$. These two measures are related by

$$
c=\frac{W}{W+(1-W) \rho_{1} / \rho_{0}}
$$

where $\rho_{0}$ and $\rho_{1}$ are the mass densities of the matrix and the fillers, respectively.

\section{RESULTS AND DISCUSSIONS}

To validate the accuracy of (1), the results were compared with experimental measurement. The underfill material considered here is an epoxy with silica fillers. Several samples with various percent weight of silica particles are tested using a TMA [3]. The measured moduli as a function of percent weight are shown in Fig. 5 (triangle symbols). Predictions of the modulus from (1) are also shown in Fig. 5 (solid line). The material constants of the matrix and filler materials used in the calculation are listed in Table I. It is seen that the agreement between the theoretically predicted and experimentally measured values is excellent. This validated the model.

\section{SUMMARY}

In this paper, a micromechanics model based on the MoriTanaka method was developed to estimate the elastic modulus of underfill materials. An explicit expression of the underfill modulus was derived as a function of filler content and the properties of the matrix and the fillers. Predictions of the modulus from this theory were compared with experimentally measured values. Excellent agreement was observed. This validated the accuracy of the model. The significance of the explicit formula for the underfill modulus is that it can be used to estimate the amount of fillers required to achieve certain modulus value for the underfill before the material is made. This reduces the amount of trial and error and in turn lowers the costs for material development.

\section{REFERENCES}

[1] R. Tummala, E. Ramaszewski, and A. Klopfenstein, Eds., Microelectronics Packaging Handbook. New York: Chapman \& Hall, 1997, vol. I, II, and III.

[2] C. A. LeGall, J. Qu, and D. McDowell, "Some mechanics issues related to the thermomechanical reliability of flip chip DCA with underfill encapsulation," Trans. ASME, vol. EEP-222, pp. 85-95, 1997.

[3] C. P. Wong, S. Shi, and G. Jefferson, "High performance no-flow underfills for low-cost flip-chip applications: material characterization," IEEE Trans. Comp., Packag., Manufact. Technol., vol. 21, pp. 450-458, Sept. 1998.

[4] C. P. Wong, M. Vincent, and S. Shi, "Fast flow and novel no flow underfills: Flow rate and CTE," ASME—Adv. Electron. Packag., vol. 19-1, pp. 301-306, 1997.

[5] T. Mura, Micromechanics of Defects in Solids. New York: Martinus Nijhoff, 1987.

[6] J. Qu, "Effects of slightly weakened interfaces on the overall elastic properties of composite materials," Mech. Mater., vol. 14, pp. 269-281, 1993.

Jianmin Qu received the B.S. degree in mathematics from Jilin University, China, and the M.S. and Ph.D. degrees in theoretical and applied mechanics from Northwestern University, Evanston, IL.

$\mathrm{He}$ is a Professor in the School of Mechanical Engineering, Georgia Institute of Technology, Atlanta. His research interest includes micromechanics of composite materials, interface mechanics, thermomechanical reliability of microelectronic packaging, and ultrasonic nondestructive evaluation and characterization. He has published over 120 papers in these areas.

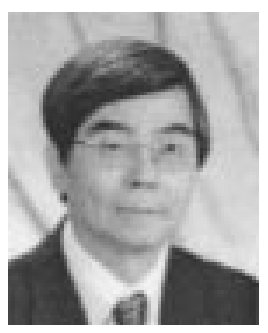

C. P. Wong (SM'87-F'92) received the B.S. degree in chemistry from Purdue University, West Lafayette, IN, and the Ph.D. degree in organic/inorganic chemistry from Pennsylvania State University, University Park.

After his doctoral study, he was awarded two years as a Postdoctoral Scholar at Stanford University, Stanford, CA. He joined AT\&T Bell Laboratories, in 1977 as Member of Technical Staff. he was elected an AT\&T Bell Laboratories Fellow in 1992. He is a Regents Professor with the School of Materials Science and Engineering and a Research Director at the NSF-funded Packaging Research Center, Georgia Institute of Technology, Atlanta. He holds over 40 U.S. patents, numerous international patents, has published over 450 technical papers and 400 keynotes and presentations in the related area. His research interests lie in the fields of polymeric materials, high Tc ceramics, materials reaction mechanism, IC encapsulation, in particular, hermetic equivalent plastic packaging, electronic manufacturing packaging processes, interfacial adhesions, PWB, SMT assembly, and components reliability.

Dr. Wong received the IEEE Components, Packaging and Manufacturing Technology (CPMT) Society Outstanding and Best Paper Awards in 1990, 1991, 1994, 1996, and 1998, the 1995 IEEE CPMT Society's Outstanding Sustained Technical Contribution Award, the 1999 Georgia Tech's Outstanding Faculty Research Program Development Award, the 1999 NSF-Packaging Research Center Faculty of the Year Award, the Georgia Tech Sigma Xi Faculty Best Research Paper Award in 2000, the University Press (London, UK) Award of Excellence in 2000 and the IEEE EAB Continuing Education Award in 2000. He served as the Technical Vice President (1990 and 1991), the President (1992 and 1993) of the IEEE CPMT Society, and was elected a member of the National Academy of Engineering in 2000. 